\title{
Field Evaluation of Fungicides, Sea Weed Biomolecules and Screening of Available Varieties/ Hybrids against Early Blight of Tomato caused by Alternaria solani
}

\author{
G. K. Sudarshan ${ }^{1 *}$, M. S. Nagaraj ${ }^{1}$, M. K. Prasanna Kumar ${ }^{1}$, \\ A. P. Mallikarjuna Gowda ${ }^{1}$, S. B. Yogananada $^{1}$ and N. Thammaiah ${ }^{2}$ \\ ${ }^{1}$ University of Agricultural Sciences, Bengaluru, Karnataka, India \\ ${ }^{2}$ University of Horticultural Sciences, Bagalkot, India \\ *Corresponding author
}

Keywords

Early blight, Alternaria solani,

Fungicides, Sea

weed biomolecules,

Screening varieties

Article Info

Accepted:

12 September 2020

Available Online:

10 October 2020

\section{A B S T R A C T}

An experiment on evaluation of fungicides, sea weed biomolecules and screening of fifty commercially available varieties/hybrids against early blight of tomato was conducted to study at ARS, Gunjevu during 2018-19. The result clearly indicates that the least disease severity of $14.33 \%$ was recorded in Hexaconazole $5 \% \mathrm{EC}$ @ $0.10 \%$ which showed significantly superior over other treatments. The next least disease severity of $15.74 \%$ was recorded in Trifloxystrobin 25 + Tebuconazole 50\%WG @ 0.07\% and which was at par with Hexaconazole 5\% EC. While, Fluxapyraxad 250 + Pyraclostrobin 250SC @ 0.05\% and Difenoconazole 25\%EC @ 0.10\% has recorded $21.70 \%$ and $23.18 \%$ disease severity respectively and were statistically at par with each other. Among the two sea weed bio molecules evaluated 39.26\% severity was recorded in Sea weed bio molecule LBD12 followed by $43.26 \%$ in Sea weed bio molecule LBD1. Among the different treatment maximum fruit yield of $57.68 \mathrm{t} /$ ha was noticed in Hexaconazole $5 \% \mathrm{EC} @ 0.1 \%$ followed by 57.19 t/ha was recorded in Trifloxystrobin $25+$ Tebuconazole $50 \%$ WG @ $0.07 \%$. The least fruit yield of $41.06 \mathrm{t} / \mathrm{ha}$ was recorded in untreated plots. The similar trends were observed in field experiment of 2019-20 and the Hexaconazole 5\%EC @ $0.1 \%$ has recorded least disease severity of $21.22 \%$ with highest yield of 58.40 t/ha. The economics of cost benefit ratio was worked for different fungicides evaluated and it was found that the highest cost benefit ratio was obtained by Hexaconazole 5\% EC (2.03) followed by Trifloxystrobin $25+$ Tebuconazole $50 \% \mathrm{WG}(1.88)$. Among the fifty commercial varieties/hybrids evaluated three varieties/ hybrids (Arka Surabh, Arka Samrat and Arka Vikas) showed moderately resistant (MR) reactions. The thirty four varieties/hybrids showed susceptible $(\mathrm{S})$ reaction and the remaining thirteen varieties/hybrids recorded highly susceptible reaction (HS).

\section{Introduction}

Alternaria solani (Ellis and martin) Jones and Grout is one of the most and frequently occurring disease on tomato and Potato in most of the parts of the world (Mathur and Shekhawat, 1986: Agrios, 2005 and Majeed et $a l ., 2014)$. The acid sweet taste and its flavour 
make it as popular vegetable among the vegetables. In India it was estimated that it was grown in an area of 0.8 Mha and 19.00 MT and productivity of 23.75 t/ha (Anonymous, 2018). Among the different Tomato growing states Andhra Pradesh, Karnataka and Madhya Pradesh together produces $40 \%$ of the country's total production. In Karnataka it grown in an area of $0.06 \mathrm{M}$ Ha with the production of $2.08 \mathrm{MT}$ and productivity of 32.40 t/ha (Anonymous, 2018).

Among the different biotic and abiotic factors, diseases were considered the main production constraints which are responsible for the reduction in yield. Among the different diseases early blight caused by Alternaria solani(Ellis and Martin) Jones and Grout is considered as important foliar disease and pathogen is a soil inhabiting air borne pathogen responsible for blight, collar rot and fruit rot of tomato. The disease in severe cases can lead to most damaging on tomato in regions with heavy dew, rainfall, high humidity and fairly high temperatures. Sufficient early blight resistance is not known within the cultivated species of tomato (Nash and Gardner, 1988). Disease effect crop production as they cause premature defoliation and results in heavy losses in production by reducing quality and quantity of fruit (Holm et al., 2003). Regarding the management of disease many workers had tried many fungicides and found that most of the Dithiocarbmates are effective in controlling the disease. Among the Dithiocarbmates Mancozeb was found effective in reducing the disease intensity and increase the yield of Pus Ruby (Kumar and Srivastava, 2013). Kumar et al., 2007., reported that Hexaconazole $(0.05 \%)$ and Azoxystrobin (0.2\%) was very effective in managing early blight of tomato.Due to continues and discriminate use fungicides, development of resistance in fungi and also increasing residual hazards has given a thrust to search for alternative method of management and also to evaluate new groups of fungicides and combination fungicides available in the market. So an attempt was made to evaluate new generation fungicides, derivatives of sea weed extracts and identification of resistance source against the pathogen of early blight of tomato.

\section{Materials and Methods}

\section{Evaluation of fungicides and sea weed extracts under field condition}

Field experiment was laid out in Randomized complete block design with ten treatments with three replication to study the efficacy of seven fungicides and two formulations of sea weed extracts against early blight was carried out during Rabi, 2018 and 2019 at ARS, Gunjevu. Twenty days old seedlings (VarietyAlnkar) were transplanted into the field with $60 \mathrm{~cm}$ inter $40 \mathrm{~cm}$ intra row spacing in plots measuring $4 \times 3 \mathrm{~m}$. All the agronomical practices and pest control measures were followed as per the recommended package of practices. The fungicides were tested as 5 sequential sprays at an interval of 10 days. The first spray was carried out as soon as the first symptom of early blight was noticed. Ten plants were randomly selected and tagged for regular observations. Observation on disease severity of foliage was recorded by using 0-9 scale as given by Mayee and Datar (1986) and per cent disease index (PDI) was worked out using formula of wheeler1969. The fruit yield in each plot was also recorded.

\section{Screening of commercial varieties/ hybrids of tomato against Alternaria solani}

A field experiment was conducted to find out the source of resistance against Alternaria blight of tomato at ARS, Gunjevu during Rabi 2018 under natural conditions. Fifty 
commercial varieties/hybrids from different seed companies and IIHR were sown with minimum of 10-12 plants per row with the spacing of $60 \times 40 \mathrm{~cm}$. The incidence on Alternaria blight under natural disease pressure was recorded.

The observations were recorded from randomly selected 5 plants in each genotypes using 0-9 scale as given by Mayee and Datar (1986). Varieties and hybrids were then grouped into five categories based on the PDI value (Mckinney, 1923) as: $<1 \%=$ immune; 1 $10 \%=$ highly resistant; $10.1-25 \%=$ resistant; 25.1-40 \% =moderately resistant; 40.1-50 $\%=$ susceptible $>50 \%=$ highly susceptible.

\section{Results and Discussion}

\section{Evaluation of Fungicides and Sea weed} extracts under field condition

The seven fungicides and two sea weed extracts were evaluated under field condition against early blight of Tomato. It is evident from the data (Table 1) that all fungicides evaluated were reduced the incidence of the disease compared to control. However rate of decrease of disease varied from fungicide to fungicide. The range of disease intensity ranged from 14.33 to $37.92 \%$ at 10 days after $5^{\text {th }}$ treatment in comparison to control $(40.22 \%)$.

The least disease severity of $14.33 \%$ was recorded in Hexaconazole 5\% EC @ 0.10\% which shows significantly superior over other treatments. The next least incidence of $15.74 \%$ was recorded in Trifloxystrobin $25+$ Tebuconazole 50\%WG @ 0.07\% and which was at par with Hexaconazole 5\%EC. The recent fungicides like Fluxapyraxad $250+$ Pyraclostrobin 250SC @ $0.05 \%$ and Difenoconazole 25\%EC @ $0.10 \%$ has recorded $21.70 \%$ and $23.18 \%$ disease severity respectively and was statistically at par with each other. Among the two weed extract evaluated $35.92 \%$ disease severity was recorded in Sea weed bio molecule LBD12 followed by $37.92 \%$ severity in Sea weed biomolecule LBD1.

Among the different treatment maximum fruit yield of $57.68 \mathrm{t} / \mathrm{ha}$ was noticed in Hexaconazole 5\%EC @ $0.1 \%$ followed by 57.19 t/ha was recorded in Trifloxystrobin 25 + Tebuconazole 50\%WG @ 0.07\%. The least fruit yield of $41.06 \mathrm{t} / \mathrm{ha}$ was recorded in untreated plots.

During the year 2019 the experiment was carried for subsequent second year and data were presented in table 2 . The results clearly indicated that among the seven fungicides evaluated against the early blight the least disease severity was observed in Hexaconazole treated plots (21.22\%) followed by Trifloxystrobin $25+$ Tebuconazole $50 \%$ WG $(22.63 \%)$ both of these fungicides were at par with each other in reducing the disease incidence. Among the other treatments tested Fluxapyraxad 250 +Pyraclostrobin 250SC @ 0.05\% has recorded $24.48 \%$ disease severity followed by Difenoconazole25\%EC @ $0.10 \%$ has recorded $26.44 \%$ disease severity.

Among the two Sea weed biomolecules evaluated Sea weed biomolecule LBD12 has showed disease severity of $41.55 \%$ followed by Sea weed biomolecule LBD1 has recorded $48.07 \%$ disease severity.

Among the fungicides and sea weed biomolecules evaluated the maximum fruit yield of 58.40 t/ha was noticed in Hexaconazole 5\%EC @ $0.1 \%$ followed by $54.84 \mathrm{t} /$ ha was recorded in Trifloxystrobin 25 + Tebuconazole 50\%WG @ 0.07\%. The least fruit yield of 40.73 t/ha was recorded in untreated plots. 
Table.1 Field evaluation of different fungicides and sea weed extracts for the management

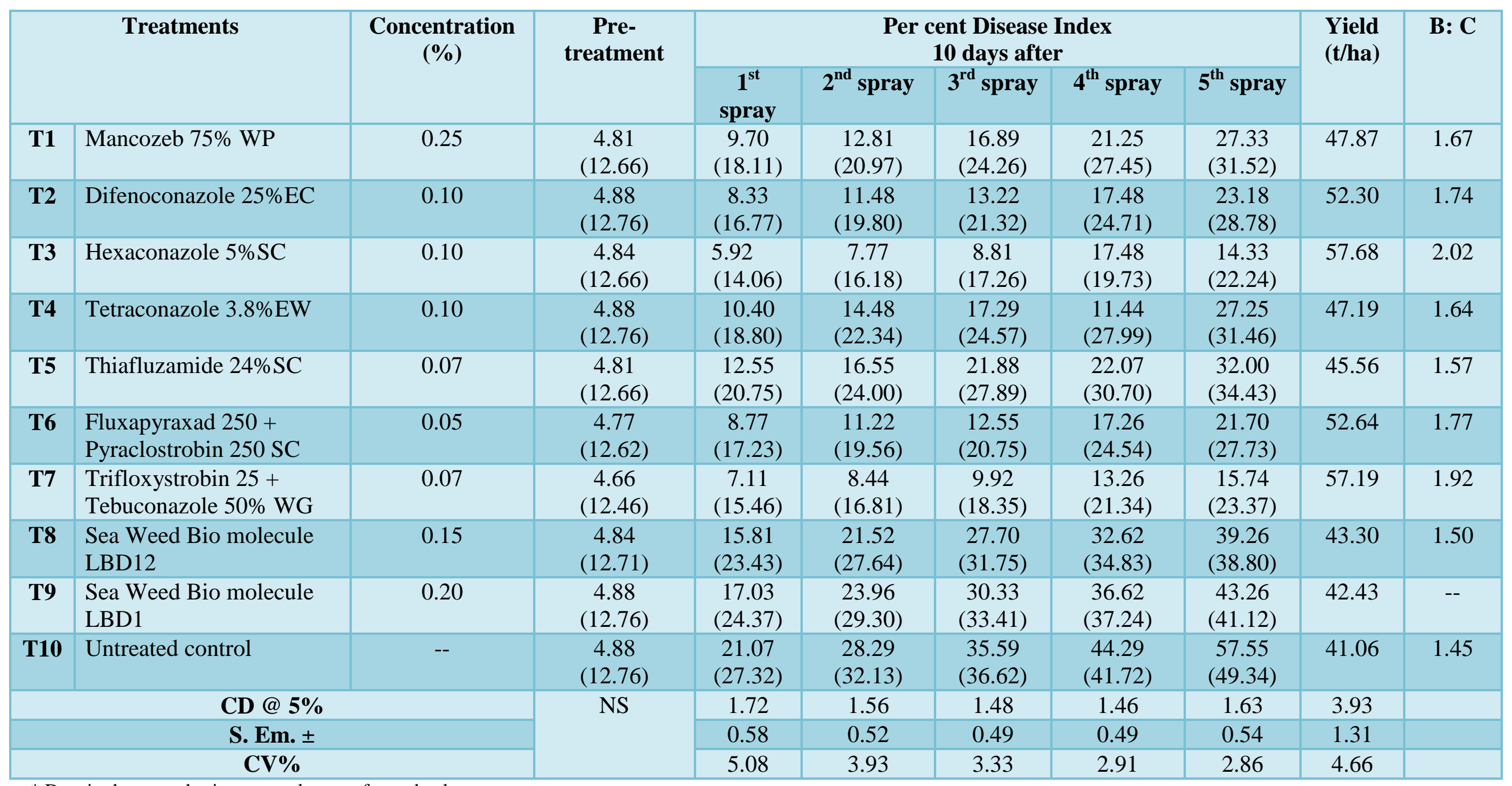

* Data in the parenthesis are angular transformed values 
Table.2 Field evaluation of different fungicides and sea weed extracts for the management of early blight of Tomato during 2019

\begin{tabular}{|c|c|c|c|c|c|c|c|c|c|c|}
\hline \multirow{2}{*}{\multicolumn{2}{|c|}{ Treatments }} & \multirow{3}{*}{$\begin{array}{c}\text { Concentratio } \\
\text { n }(\%) \\
0.25\end{array}$} & \multirow{3}{*}{$\begin{array}{c}\begin{array}{c}\text { Pre- } \\
\text { treatment }\end{array} \\
\\
6.40 \\
(14.66)\end{array}$} & \multicolumn{5}{|c|}{$\begin{array}{c}\text { Per cent Disease Index } \\
10 \text { days after }\end{array}$} & \multirow{3}{*}{$\begin{array}{r}\text { Yield } \\
\text { (t/ha) }\end{array}$} & \multirow{3}{*}{$\begin{array}{r}\text { B: } \mathbf{C} \\
\\
1.56\end{array}$} \\
\hline & & & & \multirow{2}{*}{$\begin{array}{c}\mathbf{1}^{\text {st }} \text { spray } \\
11.13 \\
(19.43)\end{array}$} & \multirow{2}{*}{$\begin{array}{c}\mathbf{2}^{\text {nd }} \\
\text { spray } \\
18.88 \\
(25.70)\end{array}$} & \multirow{2}{*}{$\begin{array}{c}\mathbf{3}^{\text {rd }} \text { spray } \\
23.26 \\
(28.80)\end{array}$} & \multirow{2}{*}{$\begin{array}{c}4^{\text {th }} \text { spray } \\
26.44 \\
(30.95)\end{array}$} & \multirow{2}{*}{$\begin{array}{c}\mathbf{5}^{\text {th }} \text { spray } \\
29.70 \\
(33.01)\end{array}$} & & \\
\hline T1 & Mancozeb 75\% WP & & & & & & & & & \\
\hline $\mathbf{T 2}$ & Difenoconazole $25 \% \mathrm{EC}$ & 0.10 & $\begin{array}{c}6.55 \\
(14.80)\end{array}$ & $\begin{array}{c}9.87 \\
(19.68)\end{array}$ & $\begin{array}{c}15.92 \\
(23.51)\end{array}$ & $\begin{array}{l}20.15 \\
(26.67)\end{array}$ & $\begin{array}{c}24.00 \\
(29.33)\end{array}$ & $\begin{array}{c}26.44 \\
(30.95)\end{array}$ & 50.63 & 1.69 \\
\hline T3 & Hexaconazole $5 \%$ SC & 0.10 & $\begin{array}{c}6.55 \\
(14.80)\end{array}$ & $\begin{array}{l}7.11 \\
(16.74)\end{array}$ & $\begin{array}{l}10.66 \\
(19.01)\end{array}$ & $\begin{array}{l}12.66 \\
(20.84)\end{array}$ & $\begin{array}{l}18.33 \\
(25.34)\end{array}$ & $\begin{array}{c}21.22 \\
(27.43)\end{array}$ & 58.40 & 2.05 \\
\hline $\mathbf{T 4}$ & Tetraconazole $3.8 \% \mathrm{EW}$ & 0.10 & $\begin{array}{c}6.55 \\
(14.80)\end{array}$ & $\begin{array}{c}11.79 \\
(21.25)\end{array}$ & $\begin{array}{l}17.66 \\
(24.83)\end{array}$ & $\begin{array}{l}21.63 \\
(27.66)\end{array}$ & $\begin{array}{l}25.70 \\
(30.43)\end{array}$ & $\begin{array}{c}30.70 \\
(33.60)\end{array}$ & 45.21 & 1.57 \\
\hline T5 & Thiafluzamide $24 \% \mathrm{SC}$ & 0.07 & $\begin{array}{c}6.55 \\
(14.80)\end{array}$ & $\begin{array}{c}13.50 \\
(22.33)\end{array}$ & $\begin{array}{l}21.18 \\
(27.38)\end{array}$ & $\begin{array}{c}25.11 \\
(30.07)\end{array}$ & $\begin{array}{c}29.33 \\
(32.79)\end{array}$ & $\begin{array}{c}36.07 \\
(36.90)\end{array}$ & 43.23 & 1.49 \\
\hline T6 & $\begin{array}{l}\text { Fluxapyraxad } 250+ \\
\text { Pyraclostrobin } 250 \text { SC }\end{array}$ & 0.05 & $\begin{array}{c}6.40 \\
(14.66)\end{array}$ & $\begin{array}{c}9.79 \\
(19.13)\end{array}$ & $\begin{array}{c}15.07 \\
(22.84)\end{array}$ & $\begin{array}{c}17.77 \\
(24.93)\end{array}$ & $\begin{array}{c}21.77 \\
(27.76)\end{array}$ & $\begin{array}{c}24.48 \\
(29.64)\end{array}$ & 50.66 & 1.71 \\
\hline $\mathbf{T 7}$ & $\begin{array}{l}\text { Trifloxystrobin } 25+ \\
\text { Tebuconazole } 50 \% \text { WG }\end{array}$ & 0.07 & $\begin{array}{c}6.55 \\
(14.83)\end{array}$ & $\begin{array}{c}8.46 \\
(18.25)\end{array}$ & $\begin{array}{l}13.83 \\
(21.39)\end{array}$ & $\begin{array}{l}14.66 \\
(22.51)\end{array}$ & $\begin{array}{l}15.85 \\
(23.46)\end{array}$ & $\begin{array}{c}22.63 \\
(28.38)\end{array}$ & 54.84 & 1.84 \\
\hline T8 & $\begin{array}{l}\text { Sea Weed Bio molecule } \\
\text { LBD12 }\end{array}$ & 0.15 & $\begin{array}{c}6.55 \\
(14.80)\end{array}$ & $\begin{array}{c}23.07 \\
(28.70)\end{array}$ & $\begin{array}{c}26.00 \\
(30.65)\end{array}$ & $\begin{array}{l}31.55 \\
(34.17)\end{array}$ & $\begin{array}{c}37.40 \\
(37.70)\end{array}$ & $\begin{array}{c}41.55 \\
(40.13)\end{array}$ & 42.79 & 1.48 \\
\hline T9 & $\begin{array}{l}\text { Sea Weed Bio molecule } \\
\text { LBD1 }\end{array}$ & 0.20 & $\begin{array}{c}6.55 \\
(14.80)\end{array}$ & $\begin{array}{c}25.18 \\
(30.12)\end{array}$ & $\begin{array}{c}27.40 \\
(31.56)\end{array}$ & $\begin{array}{c}35.77 \\
(36.73)\end{array}$ & $\begin{array}{l}42.81 \\
(40.87)\end{array}$ & $\begin{array}{c}48.07 \\
(43.89)\end{array}$ & 42.66 & -- \\
\hline T10 & Untreated control & -- & $\begin{array}{c}6.55 \\
(14.80)\end{array}$ & $\begin{array}{c}25.88 \\
(30.58)\end{array}$ & $\begin{array}{c}33.44 \\
(35.33)\end{array}$ & $\begin{array}{c}42.07 \\
(40.44)\end{array}$ & $\begin{array}{c}53.70 \\
(47.12)\end{array}$ & $\begin{array}{c}62.74 \\
(52.38)\end{array}$ & 40.73 & 1.43 \\
\hline \multicolumn{3}{|c|}{ CD@ $@ 5 \%$} & \multirow[t]{3}{*}{ NS } & 1.64 & 1.97 & 2.04 & 2.03 & 1.91 & 3.61 & \\
\hline \multicolumn{3}{|c|}{ S. Em. \pm} & & 0.55 & 0.66 & 0.68 & 0.68 & 0.64 & 1.21 & \\
\hline \multicolumn{3}{|c|}{ CV\% } & & 4.17 & 4.33 & 4.02 & 3.60 & 3.10 & 4.40 & \\
\hline
\end{tabular}

* Data in the parenthesis are angular transformed values 
Table.3 Field evaluation of different fungicides and sea weed extracts for the management of early blight of Tomato during 2018 \& 2019 (Pooled data)

\begin{tabular}{|c|c|c|c|c|c|c|c|c|c|c|}
\hline \multirow{2}{*}{\multicolumn{2}{|c|}{ Treatments }} & \multirow[t]{2}{*}{$\begin{array}{c}\text { Concentratio } \\
\text { n }(\%)\end{array}$} & \multirow[t]{2}{*}{$\begin{array}{c}\text { Pre- } \\
\text { treatment }\end{array}$} & \multicolumn{5}{|c|}{$\begin{array}{c}\text { Per cent Disease Index } \\
10 \text { days after }\end{array}$} & \multirow{2}{*}{$\begin{array}{c}\text { Yiel } \\
\text { d } \\
(\mathrm{t} / \mathrm{ha} \\
)\end{array}$} & \multirow[t]{2}{*}{ B:C } \\
\hline & & & & $1^{\text {st }}$ spray & $2^{\text {nd }}$ spray & $3^{\text {rd }}$ spray & $4^{\text {th }}$ spray & $5^{\text {th }}$ spray & & \\
\hline T1 & Mancozeb 75\% WP & 0.25 & $\begin{array}{c}5.61 \\
(13.66)\end{array}$ & $\begin{array}{c}11.13 \\
(19.43)\end{array}$ & $\begin{array}{c}15.85 \\
(23.34)\end{array}$ & $\begin{array}{c}20.07 \\
(26.53)\end{array}$ & $\begin{array}{l}23.85 \\
(29.25)\end{array}$ & $\begin{array}{c}28.51 \\
(32.47)\end{array}$ & 46.34 & 1.62 \\
\hline $\mathbf{T} 2$ & $\begin{array}{l}\text { Difenoconazole } \\
25 \% \mathrm{EC}\end{array}$ & 0.10 & $\begin{array}{c}5.72 \\
(13.80)\end{array}$ & $\begin{array}{c}9.87 \\
(18.18)\end{array}$ & $\begin{array}{l}13.70 \\
(21.66)\end{array}$ & $\begin{array}{l}16.68 \\
(23.97)\end{array}$ & $\begin{array}{c}20.74 \\
(27.02)\end{array}$ & $\begin{array}{c}24.81 \\
(29.90)\end{array}$ & 51.46 & 1.72 \\
\hline T3 & Hexaconazole $5 \%$ SC & 0.10 & $\begin{array}{c}5.70 \\
(13.73)\end{array}$ & $\begin{array}{c}7.11 \\
(15.40)\end{array}$ & $\begin{array}{c}9.22 \\
(17.60)\end{array}$ & $\begin{array}{c}10.73 \\
(19.05)\end{array}$ & $\begin{array}{c}14.88 \\
(22.65)\end{array}$ & $\begin{array}{c}17.79 \\
(24.87)\end{array}$ & 58.04 & 2.03 \\
\hline T4 & $\begin{array}{l}\text { Tetraconazole } \\
3.8 \% \mathrm{EW}\end{array}$ & 0.10 & $\begin{array}{c}5.72 \\
(13.78)\end{array}$ & $\begin{array}{l}11.79 \\
(20.02)\end{array}$ & $\begin{array}{l}16.07 \\
(23.59)\end{array}$ & $\begin{array}{l}19.46 \\
(26.12)\end{array}$ & $\begin{array}{l}23.89 \\
(28.84)\end{array}$ & $\begin{array}{l}28.98 \\
(33.04)\end{array}$ & 46.20 & 1.61 \\
\hline T5 & Thiafluzamide $24 \%$ SC & 0.07 & $\begin{array}{c}5.68 \\
(13.75)\end{array}$ & $\begin{array}{l}13.50 \\
(21.54)\end{array}$ & $\begin{array}{l}18.87 \\
(25.69)\end{array}$ & $\begin{array}{l}23.50 \\
(28.98)\end{array}$ & $\begin{array}{l}27.70 \\
(31.79)\end{array}$ & $\begin{array}{c}34.03 \\
(35.70)\end{array}$ & 44.40 & 1.53 \\
\hline T6 & $\begin{array}{c}\text { Fluxapyraxad } 250+ \\
\text { Pyraclostrobin } 250 \mathrm{SC}\end{array}$ & 0.05 & $\begin{array}{c}5.59 \\
(13.64)\end{array}$ & $\begin{array}{c}9.79 \\
(18.18)\end{array}$ & $\begin{array}{c}13.14 \\
(21.20)\end{array}$ & $\begin{array}{c}15.16 \\
(22.84)\end{array}$ & $\begin{array}{c}19.52 \\
(26.59)\end{array}$ & $\begin{array}{c}23.09 \\
(28.94)\end{array}$ & 51.65 & 1.74 \\
\hline $\mathbf{T 7}$ & $\begin{array}{c}\text { Trifloxystrobin } 25+ \\
\text { Tebuconazole } 50 \% \\
\text { WG }\end{array}$ & 0.07 & $\begin{array}{c}5.61 \\
(13.65)\end{array}$ & $\begin{array}{c}8.46 \\
(16.86)\end{array}$ & $\begin{array}{c}10.88 \\
(19.10)\end{array}$ & $\begin{array}{c}12.29 \\
(20.43)\end{array}$ & $\begin{array}{c}14.55 \\
(22.41)\end{array}$ & $\begin{array}{c}19.18 \\
(26.17)\end{array}$ & 56.01 & 1.88 \\
\hline T8 & $\begin{array}{l}\text { Sea Weed Bio } \\
\text { molecule LBD12 }\end{array}$ & 0.15 & $\begin{array}{c}5.70 \\
(13.76)\end{array}$ & $\begin{array}{c}19.44 \\
(26.06)\end{array}$ & $\begin{array}{c}22.29 \\
(29.14)\end{array}$ & $\begin{array}{c}29.63 \\
(32.96)\end{array}$ & $\begin{array}{c}35.01 \\
(36.27)\end{array}$ & $\begin{array}{c}40.40 \\
(39.47)\end{array}$ & 43.05 & 1.49 \\
\hline T9 & $\begin{array}{l}\text { Sea Weed Bio } \\
\text { molecule LBD1 }\end{array}$ & 0.20 & $\begin{array}{c}5.72 \\
(13.80)\end{array}$ & $\begin{array}{c}21.11 \\
(27.25)\end{array}$ & $\begin{array}{c}24.57 \\
(30.43)\end{array}$ & $\begin{array}{c}33.05 \\
(35.07)\end{array}$ & $\begin{array}{c}39.72 \\
(39.05)\end{array}$ & $\begin{array}{c}45.66 \\
(42.51)\end{array}$ & 42.54 & -- \\
\hline T10 & Untreated control & -- & $\begin{array}{c}5.72 \\
(13.80)\end{array}$ & $\begin{array}{c}23.48 \\
(28.95)\end{array}$ & $\begin{array}{c}27.09 \\
(33.73)\end{array}$ & $\begin{array}{c}38.83 \\
(38.53)\end{array}$ & $\begin{array}{c}49.00 \\
(44.42)\end{array}$ & $\begin{array}{c}60.14 \\
(50.86)\end{array}$ & 40.89 & 1.44 \\
\hline \multicolumn{3}{|c|}{ CD@ $@ 5 \%$} & \multirow{3}{*}{ NS } & 1.13 & 1.24 & 1.17 & 1.38 & 1.31 & 2.48 & \\
\hline \multicolumn{3}{|c|}{ S. Em. \pm} & & 0.40 & 0.43 & 0.41 & 0.48 & 0.46 & 0.87 & \\
\hline \multicolumn{3}{|c|}{ CV\% } & & 4.57 & 4.31 & 3.66 & 3.84 & 3.27 & 4.42 & \\
\hline
\end{tabular}

* Data in the parenthesis are angular transformed values 
Table.4 Screening of tomato varieties and hybrids against early blight caused by Alternaria solani under natural epiphytotic conditions

\begin{tabular}{|c|c|c|c|c|}
\hline \multirow[t]{2}{*}{ SI. No. } & \multirow[t]{2}{*}{ Variety/ Hybrid } & \multicolumn{2}{|c|}{ Per cent disease Index } & \multirow[t]{2}{*}{ Disease reaction } \\
\hline & & Flowering stage & Fruit Harvesting stage & \\
\hline 1 & Arka Alok & 8.66 & 49.77 & $\mathrm{~S}$ \\
\hline 2 & Arka Saurabh & 8.88 & 34.44 & MR \\
\hline 3 & ArkaMeghali & 8.88 & 48.00 & $\mathrm{~S}$ \\
\hline 4 & Arka Samrat & 8.44 & 37.33 & MR \\
\hline 5 & ArkaRakshak & 18.66 & 49.33 & $\mathrm{~S}$ \\
\hline 6 & ArkaAabha & 10.44 & 48.44 & $\mathrm{~S}$ \\
\hline 7 & Arka Vikas & 16.88 & 39.55 & MR \\
\hline 8 & Emerald & 9.33 & 40.88 & $\mathrm{~S}$ \\
\hline 9 & NS-538 & 16.22 & 46.66 & $\mathrm{~S}$ \\
\hline 10 & NS-501 & 7.77 & 48.88 & $\mathrm{~S}$ \\
\hline 11 & NS-5002 & 8.66 & 41.77 & $\mathrm{~S}$ \\
\hline 12 & Abhilash & 9.33 & 43.11 & $\mathrm{~S}$ \\
\hline 13 & Shivam & 8.44 & 49.33 & S \\
\hline 14 & SYN-1057 & 9.55 & 40.88 & $\mathrm{~S}$ \\
\hline 15 & Sampurna & 9.33 & 48.88 & $\mathrm{~S}$ \\
\hline 16 & Abhinav & 27.55 & 49.77 & $\mathrm{~S}$ \\
\hline 17 & Rishika & 18.88 & 58.66 & HS \\
\hline 18 & Meghdout & 9.77 & 56.44 & HS \\
\hline 19 & NS-629 & 19.55 & 45.77 & $\mathrm{~S}$ \\
\hline 20 & JK-811 & 8.66 & 48.88 & $\mathrm{~S}$ \\
\hline 21 & US-4722 & 8.00 & 41.77 & $\mathrm{~S}$ \\
\hline 22 & US-1143 & 9.33 & 43.11 & $\mathrm{~S}$ \\
\hline 23 & US-440 & 9.55 & 40.88 & $\mathrm{~S}$ \\
\hline 24 & HMC-01 & 9.77 & 48.88 & $\mathrm{~S}$ \\
\hline 25 & HMC-02 & 9.77 & 49.77 & $\mathrm{~S}$ \\
\hline 26 & HMC-03 & 10.00 & 47.11 & $\mathrm{~S}$ \\
\hline 27 & HMC-04 & 18.00 & 48.11 & S \\
\hline 28 & NCSTMH-01 & 18.44 & 48.88 & $\mathrm{~S}$ \\
\hline 29 & NCSTMH-02 & 18.88 & 50.22 & HS \\
\hline 30 & NCSTMH-03 & 7.11 & 51.11 & HS \\
\hline 31 & NCSTMH-04 & 9.11 & 49.77 & $\mathrm{~S}$ \\
\hline 32 & NCSTMH-05 & 18.44 & 59.11 & HS \\
\hline 33 & NCSTMH-06 & 19.11 & 60.00 & HS \\
\hline 34 & NCSTMH-07 & 19.55 & 60.88 & HS \\
\hline 35 & NCSTMH-08 & 18.66 & 48.44 & $\mathrm{~S}$ \\
\hline 36 & NCSTMH-09 & 17.77 & 61.33 & HS \\
\hline 37 & NCSTMH-10 & 19.33 & 64.44 & HS \\
\hline 38 & NCSTMH-11 & 17.55 & 50.66 & $\mathrm{~S}$ \\
\hline 39 & NCSTMH-12 & 9.11 & 49.77 & $S$ \\
\hline 40 & NCSTMH-13 & 9.55 & 49.77 & $\mathrm{~S}$ \\
\hline 41 & NCSTMH-14 & 10.44 & 48.44 & $\mathrm{~S}$ \\
\hline 42 & NCSTMH-15 & 10.22 & 50.66 & HS \\
\hline 43 & NCSTMH-16 & 18.44 & 48.88 & $\mathrm{~S}$ \\
\hline 44 & NCSTMH-17 & 18.00 & 49.33 & $\mathrm{~S}$ \\
\hline 45 & NCSTMH-18 & 18.66 & 47.55 & $\mathrm{~S}$ \\
\hline 46 & NCSTMH-19 & 19.11 & 50.22 & HS \\
\hline 47 & NCSTMH-20 & 19.33 & 51.11 & HS \\
\hline 48 & NCSTMH-21 & 18.88 & 41.11 & $\mathrm{~S}$ \\
\hline 49 & NCSTMH-22 & 17.55 & 42.00 & $\mathrm{~S}$ \\
\hline 50 & Alankar & 20.66 & 62.22 & HS \\
\hline
\end{tabular}


Table.5 Disease reaction of varieties and hybrids in terms of per cent disease index

\begin{tabular}{|c|c|c|}
\hline PDI Scale & Disease reaction & Varieties/ hybrids \\
\hline $\mathbf{1 1 \%}$ & Immune & - \\
\hline $\mathbf{1 - 1 0 \%}$ & Highly Resistant & - \\
\hline $\mathbf{1 0 . 1 - 2 5 \%}$ & Resistant & Arka Saurabh, Arka Samrat, Arka Vikas \\
\hline $\mathbf{2 5 . 1 - 4 0 \%}$ & Moderately Resistant & Arka Alok, ArkaMeghali, ArkaRakshak. Arkaabha, \\
\hline $\mathbf{4 0 . 1 - 5 0 \%}$ & Susceptible & Emerald, NS-538, NS-501, NS-5002, Abhilash, \\
& & Shivam, SYN-1057, Sampurna, Abhinav, NS-629, \\
& JK-811, US-4722, US-1143,US-440,HMC-01, \\
& & HMC-02,HMC-03,HMC-04, NCSTMH-01, \\
& NCSTMH-04, NCSTMH-08, NCSTMH-11, \\
& & NCSTMH-12, NCSTMH-13, NCSTMH-14, \\
& NCSTMH-16, NCSTMH-17, NCSTMH-18, \\
& Highly susceptible & NishCSTMH-21, NCSTMH-22 \\
\hline $\mathbf{5 0 \%}$ & & NCSTMH-05, NCSTMH-06, NCSTMH-07,, \\
& & NCSTMH-09, NCSTMH-10, NCSTMH-15, \\
& & NCSTMH-19, NCSTMH-20, Alnkar \\
\hline
\end{tabular}

Two years pooled data (2018 \& 2019) was presented in Table 3 clearly revealed that among the fungicides and sea weed biomolecules evaluated all the treatments showed superiority over control in reducing the incidence of disease. Among the treatments disease intensity ranged from $17.79 \%$ to $45.66 \%$ as against $60.14 \%$ in untreated control. The least disease severity of $17.79 \%$ was recorded in Hexaconazole 5\%EC (a) $0.1 \%$ followed by $19.18 \%$ disease severity in Trifloxystrobin $25+$ Tebuconazole $50 \%$ WG @ $0.07 \%$ which were at par with each other in reducing the disease severity. Among the other fungicides evaluated Fluxapyraxad 250 +Pyraclostrobin 250SC @ $0.05 \%$ has showed significantly superior in reducing the disease severity and recorded $23.09 \%$ followed by Difenoconazole $25 \%$ EC (a) $0.1 \%(24.81 \%)$ and both of these fungicides were at par with each other in reducing the disease severity. The maximum fruit yield of $58.04 \mathrm{t} / \mathrm{ha}$ was recorded in Hexaconazole 5\% EC followed by $56.01 \mathrm{t} / \mathrm{ha}$ was recorded in Trifloxystrobin $25+$ Tebuconazole $50 \%$ WG.
Among the different fungicides Fluxapyraxad $250+$ Pyraclostrobin 250 SC and Difenoconazole25\%EC has recorded fruit yield of $51.65 \mathrm{t} / \mathrm{ha}$ and $51.46 \mathrm{t} / \mathrm{ha}$ respectively. Economics of cost benefit ratio of pooled data clearly indicates that spraying of Hexaconazole 5\%EC @ 0.1\% will bring the highest returns compared to other treatments with the highest cost benefit ratio of 2.03 followed by cost benefit ratio of 1.88 was obtained in Trifloxystrobin $25+$ Tebuconazole $50 \% \mathrm{WG}$. The present results are in conformity with the earlier workers. Palaiah et al., (2020) found that Azoxystrobin $11 \%$ + Tebuconazole 18.3\% SC @ 1000ml /ha was most effective fungicide for controlling the early blight of tomato with maximum fruit yield of $41.83 \mathrm{t} / \mathrm{ha}$. Arun kumar et al., (2013) found that Propiconazole $(0.1 \%)$ and Pyraclostrobin $(0.2 \%)$ were most effective in reducing severity of the disease and increasing fruit yield over control. Bartlett et al., (2002) evaluated effect of different Strobilurins fungicides like Azoxystrobin, Kresoxim methyl, 
Trifloxystrobin and Pyraclostrobin on quality and yield of Barley, wheat, tomato, potato and mangoes and reported that spraying of Strobilurins influenced in increasing the yield compared to trizoles.

Screening of commercial hybrids and varieties of tomato against Alternaria solani

In this experiment disease observations were recorded under natural epiphytotic conditions during flowering and fruit harvesting stage. From the table 4 it is clearly shows that reactions varied with varieties / hybrids. The mean disease intensity during flowering stage ranged from $7.11 \%$ to $20.66 \%$ and during fruit harvesting stage the disease intensity was ranged from $34.44 \%-64.44 \%$. Out of fifty varieties / hybrids evaluated three varieties/ hybrids namely Arka Surabh, Arka Samrat and Arka Vikas showed moderately resistant (MR) reactions. The thirty four varieties/ hybrids viz., Arka Alok, Arka Meghali, Arka Rakshak. Arka Abha, Emerald, NS-538, NS501, NS-5002, Abhilash, Shivam, SYN-1057, Sampurna, Abhinav, NS-629, JK-811, US4722, US-1143, US-440, HMC-01, HMC02,HMC-03, HMC-04, NCSTMH-01, NCSTMH-04, NCSTMH-08, NCSTMH-11, NCSTMH-12, NCSTMH-13, NCSTMH-14, NCSTMH-16, NCSTMH-17, NCSTMH-18, NCSTMH-21, NCSTMH-22 showed susceptible (S) reaction and remaining thirteen varieties and hybrids namely Rishika, Meghdout, NCSTMH-02, NCSTMH-03, NCSTMH-05, NCSTMH-06, NCSTMH-07, NCSTMH-09, NCSTMH-10, NCSTMH-15, NCSTMH-19, NCSTMH-20, Alankar has recorded highly susceptible reaction(HS). Kumar and Srivastava (2013) screened forty four genotypes and the genotype EC-521071 was found resistant fifteen genotypes (Floraded, Kashi Sharad, CO-3, Punjab Upma, TLC- 1, NDTVR-60, Selection-7, BT120, Suncherry, Swarna Naveen, Cholnak-K, T. Local, EC-521086, EC-521069 and
EC531803) showed moderately resistant and twenty genotypes (Pusa Sadabahar, DT-2, Pant T-3, H-24, H-86, N D T-3, Selection- 18, VR-20, Azad T-5, Sworn Lalima, Flawery, Feb-4, NF-315, Kajela, Angur Lata, Columbia, Grant, P.M S-1, Superbug andShalimar-2 showed susceptible and five genotypes (PS-1, Kashi Amrit, Fla-7171, HT-4 and DT-10) were found highly susceptible and two genotypes H-88-74-1 and EC-520061 was found highly resistant to early blight of tomato. Majeed et al., 2020 conducted experiment to screen 50 genotypes under field condition. Among these Germplasms, 07 test lines viz., 2014/ TODHYB-7, 2015/TODHYB-2, 2016/ TODHYB-4, 2016/TODHYB-6, 2016/ TODHYB-7, 2016/TODVAR-10 and 2016/ TODVAR-12 exhibited complete resistant against early blight and 14 lines viz., 2014/ TODHYB-1, 2014/TODVAR-3， 2014/ TODVAR-1, 2014/TODVAR-4, 2014/ TODVAR-6, 2015/TODVAR-1, 2016/ TODVAR-3, 2016/ TODVAR-4, 2016/ TODVAR-6, 2016/TODVAR-7, 2016/ TODVAR-8, 2015/ TOINDVAR-2, 2015/ TOINDVAR-3 and 2015/TOINDVAR-5 were found moderately resistant (Table 5).

\section{Acknowledgement:}

I thank M/s HM Clause India Pvt Ltd., M/s Nirmal Seeds, M/s Namdhari seeds Pvt. Ltd., M/s Nunhemps seeds , M/s Syngenta India, for providing seed material for research purpose.

\section{References}

Agrio, G.N. 2005, Plant Pathology. (5 edition). Academic press. 922pp.

Anonymous, 2018, Horticultural statistics at a glance 2018, Pub: Ministry of Agriculture and farmer welfare, GOI, 458.

Arunkumar, K.T., Kulkarni, M.S., 
Thammaiah, N. and Hegde, Y., 2010, Fungicidal management of early blight of tomato. Indian phytopathol. 63(1): 96-97.

Barlett, D.W., Clough, J.M., Godwin, J.R., HALL, A.A., Hamer, M. and Parrdobrzanski, B., 2002, The strobilurin fungicides. Pest Manag. Sci. 58: 649662.

Holm, A.L., Rivera, V.V., Secor, G.A. and Gudmestad, N.C., 2003, Temporal sensitivity of Alternaria solani to foliar fungicides (short communication). Amer. J.of Potato Research. 80: 33-40.

Kumar, S. and Srivastava. K., 2013, Screening of Tomato genotypes against early blight Alternaria solani under field conditions. The Bioscan. 8(1): 189-193.

Kumar, V., Gupta, R.C., Singh, P.C., Pandey, K.K., Kumar, R., Rai, A.S.and Rai, M., 2007, Management of early blight disease of tomato cv. Kashi Amrit through fungicides, bioagents and cultural practices in India. Veg. Sci. 34(2):206-207.

Majeed, A., Ahmad, H. and Khan, H., 2014, Effect of systemic and contact fungicides on late blight disease and tuber yield of potato. Journal of Agricultural technology. 10(1): 209217.

Majeed, M., Badr, Z.A., Bhat, Z.A., Bhat, M.A., Chattoo, M.A., Makhdhoomi,
M.I. and Zargar, S.M., 2020, Screening of tomato genotypes against early leaf blight (Alternaria solani). Int. J. Curr. Microbiol. App. Sci. 9 (1): 1140-1147.

Mathur, K. and Shekhawat, K.S., 1986, Chemical control of early blight in kharif sown tomato. Indian J. Mycol. Pl. Pathol. 16: 235-238.

Mayee, C.D. and Datar, V.V., 1986, Phytopathometry, Marathwad Agricultural University, Parabhani, p.95.

Mckinney, H.H., 1923, Influence of soil temperature and moisture on infection of wheat seedlings by Helminthosporium sativum. J. Agri, Res. 26: 195-217.

Nash, A.F. and Gardner, R.G., 1988, Heritability of tomato early blight resistance derived from Lycopersicon hirsutum P.I.126445. J.Am.Soc. Hort.Sci. 113: 264-268.

Palaiah, P., Vinay, J.U., Vinay Kumar, H.D. and Shiva Kumar, K.V., 2020, Management of early blight of tomato (Alternaria solani) through new generation fungicides under field condition. Int. J. Chem. Stu., 8: 11931195.

Wheeler, Bej, 1969, Pathometric calculations. In an Introduction to plan Diseases. John wiley and sons. Ltd. London.301.

\section{How to cite this article:}

Sudarshan, G. K., M. S. Nagaraj, M. K. Prasanna Kumar, A. P. Mallikarjuna Gowda, S. B. Yogananada and Thammaiah, N. 2020. Field Evaluation of Fungicides, Sea Weed Biomolecules and Screening of Available Varieties/ Hybrids against Early Blight of Tomato caused by Alternaria solani. Int.J.Curr.Microbiol.App.Sci. 9(10): 1484-1493. doi: https://doi.org/10.20546/ijcmas.2020.910.177 